\title{
Endomorphisms and Product Bases of the Baer-Specker Group
}

\author{
E. F. Cornelius Jr. \\ College of Engineering and Science, University of Detroit Mercy, Detroit, MI 48221-3038, USA \\ Correspondence should be addressed to E. F. Cornelius Jr., efcornelius@comcast.net
}

Received 31 March 2009; Revised 29 July 2009; Accepted 12 August 2009

Recommended by Dorothy Wallace

The endomorphism ring of the group of all sequences of integers, the Baer-Specker group, is isomorphic to the ring of row finite infinite matrices over the integers. The product bases of that group are represented by the multiplicative group of invertible elements in that matrix ring. All products in the Baer-Specker group are characterized, and a lemma of László Fuchs regarding such products is revisited.

Copyright @ 2009 E. F. Cornelius Jr. This is an open access article distributed under the Creative Commons Attribution License, which permits unrestricted use, distribution, and reproduction in any medium, provided the original work is properly cited.

\section{Introduction}

Abelian group notation and terminology are standard $[1,2] . \mathbb{N}$ denotes the nonnegative integers and $\mathbb{N}^{+}$the positive integers. $\mathbb{Z}$ denotes the ring of all integers. Let $\mathbf{P}=\mathbb{Z}^{\mathbb{N}}=\prod_{\mathbb{N}} \mathbb{Z}$, the group of all integer sequences, known as the Baer-Specker group [3, 4]. For purposes of matrix vector multiplication, an element $\mathbf{x}$ of $\mathbf{P}$ is viewed as a column vector, the $i$ th entry of which is denoted $x_{i}$.

\section{Infinite Integral Matrices and Their Operations}

To prove the results claimed, some properties of infinite integral matrices are needed. These properties are stated without proof, as they are known or easily proved.

The group of all infinite integral matrices is denoted by $\mathcal{M}$, with addition being defined in the usual way. Matrix rows and columns are indexed by $\mathbb{N}$. Column operations (multiplying a column by -1 , interchanging two columns, adding an integral multiple of one column to another) are carried out in the usual way.

$F \in \mathcal{M}$ is said to be row finite if, for each $i \in \mathbb{N}, F_{i j}=0$ for all but finitely many $j \in \mathbb{N}$; that is, if for each $i$, there exists $k_{i} \in \mathbb{N}$ such that $F_{i j}=0$ unless $j \leq k_{i}$. A column finite matrix 
is defined analogously. The additive group of all row finite matrices in $\mathcal{M}$ is denoted by $\mathcal{F}$. $T \in \mathcal{M}$ is said to be lower triangular if $T_{i j}=0$ unless $i \geq j$. The additive group of all lower triangular matrices in $\mathcal{M}$ is denoted by $\tau$. Obviously $\tau \subset \mathcal{F}$.

Recall that multiplication of two infinite matrices may not be well defined and, even when defined, may not be associative. For examples of pathologies of infinite matrices, see Section 5. However, for $F \in \mathcal{F}$ and $G \in \mathcal{M}$, the product of $F$ and $G$, denoted $F G \in \mathcal{M}$, is defined as

$$
F G_{i j}=\sum_{k \in \mathbb{N}} F_{i k} G_{k j}, \quad i, j \in \mathbb{N} .
$$

Because $F$ is row finite, the sum reduces to $\sum_{k \leq n_{i}} F_{i k} G_{k j}$ where $n_{i} \in \mathbb{N}$. Under multiplication thus defined, $\mathcal{F}$ and $\tau$ are rings with identity $I$ satisfying $I_{i j}=\delta_{i j}$, the Kronecker delta.

$F \in \mathcal{F}$ is said to be invertible if there exists $G \in \mathcal{F}$ satisfying $F G=I=G F$. It clear that such a $G$ is a unique two-sided inverse, so that $F^{-1}=G$ is well defined [5, pages 21-25]. The set of invertible matrices in $\mathcal{F}$ is denoted $\mathcal{G} \perp$ and forms a group under matrix multiplication.

Proposition 2.1. If $F^{\prime} \in \mathcal{F}$ is obtained from $F \in \mathcal{F}$ through a column operation, then $F^{\prime}=F E$ where $E$ is obtained by performing the same operation on $I . E$ is both row and column finite and invertible.

This corollary is immediate.

Corollary 2.2. If $F^{\prime} \in \mathcal{F}$ is obtained from $F \in \mathcal{F}$ through a finite sequence of column operations, then $F^{\prime}=F E$ where $E$ is obtained by performing the same sequence of operations on $I$. E is both row and column finite and invertible.

Let $U=\left\{\mathbf{u}_{j}: j \in \mathbb{N}\right\}$ be a subset of $\mathbf{P}$. If each expression $\sum_{j \in \mathbb{N}} b_{j} \mathbf{u}_{j}, b_{j} \in \mathbb{Z}$, represents an element of $\mathbf{P}$, then the set of all such elements forms a subgroup of $\mathbf{P}$, denoted $\prod_{j \in \mathbb{N}}\left\langle\mathbf{u}_{j}\right\rangle$ and called a product in $\mathbf{P}$ [2, page 164]. Such a subset $U$ forms a product if and only if the matrix with columns $\mathbf{u}_{j}$ is row finite. If the representations are unique, then the $\mathbf{u}_{j}$ are independent and $U$ is said to be a basis of the product. One of the objectives of this paper is to characterize products and product bases in term of endomorphisms of $\mathbf{P}$.

For $F \in \mathcal{F}$ and $\mathbf{x} \in \mathbf{P}$, the matrix vector product of $F$ and $\mathbf{x}$, denoted simply $F \mathbf{x}$, is defined as $F \mathbf{x} \in \mathbf{P}$, with $F \mathbf{x}_{i}=\sum_{k \in \mathbb{N}} F_{i k} x_{k}, i \in \mathbb{N}$. As with matrix multiplication, because $F$ is row finite, the sum reduces to $\sum_{k \leq n_{i}} F_{i k} x_{k}$ where $n_{i} \in \mathbb{N}$. Each such $F$ naturally induces an endomorphism of $\mathbf{P}$, via matrix vector multiplication. Indeed, a product in $\mathbf{P}, \prod_{j \in \mathbb{N}}\left\langle\mathbf{u}_{j}\right\rangle$, is simply the image of such an endomorphism, where the matrix $F$ is constructed with columns $\mathbf{u}_{j}, j \in \mathbb{N}$.

All endomorphisms of $\mathbf{P}$ are determined by their values on $\boldsymbol{\Sigma}$, the free subgroup of $\mathbf{P}$ consisting of sequences which are 0 after awhile [2, Lemma 94.1]. The standard group basis of $\boldsymbol{\Sigma}$ is $\left\{\mathbf{e}_{j}:\left(\mathbf{e}_{j}\right)_{i}=\delta_{i j}, i, j \in \mathbb{N}\right\}$, and the $\mathbf{e}_{j}$ also form the standard product basis of $\mathbf{P}$. In particular, all endomorphisms of $\mathbf{P}$ are determined by their values on this standard basis. As a result, with each element $f$ in the endomorphism ring of $\mathbf{P}, \operatorname{End}(\mathbf{P})$, is associated a unique matrix $M^{f} \in \mathcal{M}$, given by $M_{i j}^{f}=f\left(\mathbf{e}_{j}\right)_{i}, i, j \in \mathbb{N}$. The immediate goal is to prove that each such $M^{f}$ is row finite, so that every endomorphism of $\mathbf{P}$ is effectively multiplication by a row finite matrix. 


\section{Endomorphisms and Product Bases}

The main theorem follows.

Theorem 3.1. Every endomorphism of $\mathbf{P}$ is induced by the action of a row finite, infinite, integral matrix.

Proof. Let $f \in \operatorname{End}(\mathbf{P})$, and let $M^{f} \in \mathcal{M}$ be the matrix associated with $f, M_{i j}^{f}=f\left(\mathbf{e}_{j}\right)_{i}, i, j \in \mathbb{N}$. Suppose that $M^{f}$ is not row finite; that is, suppose there are $i$ and distinct $j_{k} \in \mathbb{N}$ such that $M_{i j_{k}}^{f} \neq 0$ for all $k \in \mathbb{N}$. Let $\pi_{i}$ denote the canonical projection of the $i$ th row of elements of $\mathbf{P}$ onto $\mathbb{Z}$. Then $\pi_{i} f$ would be a homomorphism of $\mathbf{P}$ into $\mathbb{Z}$, which is nonzero at all $\mathbf{e}_{j_{k}}, k \in \mathbb{N}$, an impossibility [4, Satz III]. Hence it is not possible that an infinite number of the $M_{i j}^{f}$ are nonzero; that is, $M_{f}$ must be row finite.

To see that $M_{f}$ acts on $\mathbf{P}$ to produce $f$, it suffices to check agreement on the standard basis of $\boldsymbol{\Sigma}$. For $i, j \in \mathbb{N},\left(M^{f} \mathbf{e}_{j}\right)_{i}=\sum_{k \in \mathbb{N}} M_{i k}^{f}\left(\mathbf{e}_{j}\right)_{k}=\sum_{k \in \mathbb{N}} f\left(\mathbf{e}_{k}\right)_{i} \delta_{j k}=f\left(\mathbf{e}_{j}\right)_{i}$.

Corollary 3.2. (i) The endomorphism ring of $\mathbf{P}, \operatorname{End}(\mathbf{P})$, is isomorphic to the ring of row finite, infinite, integral matrices $\mathbf{F}$. (ii) The automorphism group of $\mathbf{P}, A u t(\mathbf{P})$, is isomorphic to the multiplicative group of invertible matrices in $\mathcal{F}, \mathcal{G} \mathcal{\perp}$.

Proof. For $f \in \operatorname{End}(\mathbf{P}), f \rightarrow M^{f}$ is a ring isomorphism with $\mathcal{F}$, which maps $\operatorname{Aut}(\mathbf{P})$ isomorphically onto $\mathcal{G} \mathcal{\perp}$. The row finiteness of the elements of $\mathcal{F}$ ensures that all sums are finite and that matrix multiplication is associative.

Corollary 3.3. There is a one-to-one correspondence between the matrices of $\mathcal{G} \perp$ and the product bases of $\mathbf{P}$.

Proof. Let $\left\{\mathbf{u}_{j}: j \in \mathbb{N}\right\}$ be a product basis for $\mathbf{P}$, and let $A \in A u t(\mathbf{P})$ be defined by $A(\mathbf{x})_{i}=a_{i}$ for $\mathbf{x} \in \mathbf{P}$, when $\mathbf{x}=\sum_{j \in \mathbb{N}} a_{j} \mathbf{u}_{j}, a_{j} \in \mathbb{Z}$. The uniqueness of expression of the elements of $\mathbf{P}$ in terms of the product basis guarantees that $A$ is an automorphism. By Corollary 3.2(ii), $A$ corresponds to a unique element of $\mathcal{G} \mathcal{L}$. The converse is clear.

\section{Products in $\mathbf{P}$}

Topological techniques are helpful in studying the endomorphisms of $\mathbf{P}$. Background material may be found in [6-8] and the references cited therein. For $\mathbf{x} \neq \mathbf{y} \in \mathbf{P}$, the distance between them, $d(\mathbf{x}, \mathbf{y})$, is defined to be $2^{-n}$, where $n$ is the first place at which $x_{n} \neq y_{n}$. Of course, $d(\mathbf{x}, \mathbf{x})=0$. It is easy to check that $d$ is a metric on $\mathbf{P}$, which comports with the product topology on $\mathbf{P}$, when $\mathbb{Z}$ is discrete.

$\mathbf{P}$ is a separable metric space having $\boldsymbol{\Sigma}$ as a countable dense subset. Finally, $\mathbf{P}$ is a complete metric space in which Cauchy sequences eventually become constant pointwise.

The important aspect of this topology is that all endomorphisms of $\mathbf{P}$ are continuous.

Similarly, $\mathcal{M}$ is a complete separable metric space with the distance between distinct matrices $F$ and $G$ defined as $2^{-n}$, where $n=\min \left\{i, j: F_{i j} \neq G_{i j}\right\}$.

Theorem 4.1. Every product in $\mathbf{P}$ can be generated by a lower triangular matrix, the columns of which form a basis of the product. 
Proof. Let $F \in \mathcal{F}$ generate the product $F \mathbf{P}$. The proof proceeds by transforming $F$ via column operations into a lower triangular matrix $T$ satisfying $T \mathbf{P}=F \mathbf{P}$. Since $F=0$ is trivial, assume $F \neq 0$. Let $\mathbf{y} \in F \mathbf{P}, \mathbf{0} \neq \mathbf{y}=\left(y_{0}, y_{1}, \ldots\right)^{t}$, where $t$ denotes transpose, and suppose $\mathbf{y}=F \mathbf{x}$ for $\mathbf{x}=\left(x_{0}, x_{1}, \ldots\right)^{t} \in \mathbf{P}$.

If row 0 of $F$ consists entirely of $0^{\prime}$ s, simply set $T^{1}=F, E^{1}=I$, and $\mathbf{x}^{1}=\mathbf{x}$ and proceed to the next step. Otherwise, perform column operations on the nonzero entries of row 0 of $F$ to obtain a new matrix $T^{1}$ in which $T_{0 j}^{1}=0$ for all $j>0$. This is tantamount to finding the gcd of the entries in row 0 , columns $j \geq 0$ of $F$. Perform the same column operations on $I$ to produce $E^{1}$, so that $T^{1}=F E^{1}$ with $E^{1}$ an invertible row and column finite matrix. Let $G^{1}$ denote the inverse of $E^{1}$. Since $F=T^{1} G^{1}, \mathbf{y}=F \mathbf{x}=T^{1}\left(G^{1} \mathbf{x}\right)$. Set $\mathbf{x}^{1}=G^{1} \mathbf{x}=\left(x_{0}^{1}, x_{1}^{1}, \ldots\right)^{t}$ so that $\mathbf{y}=T^{1} \mathbf{x}^{1}$.

If $T_{1 j}^{1}=0$ for all $j \geq 1$, simply set $T^{2}=T^{1}, \mathbf{x}^{2}=\mathbf{x}^{1}$, and $E^{2}=I$ and proceed. Otherwise, perform column operations on the nonzero entries of row 1 in columns $j \geq 1$ of $T^{1}$ to obtain a new matrix $T^{2}$ in which $T_{1 j}^{2}=0$ for all $j>1$. As before, this is tantamount to finding the gcd of the entries in row 1 , columns $j \geq 1$ of $T^{1}$. Note that $T_{i 0}^{2}=T_{i 0}^{1}$ for all $i$; that is, column 0 of $T^{2}$ is the same as column 0 of $T^{1}$.

Perform the same column operations on $I$ to obtain $E^{2}$, so that $T^{2}=T^{1} E^{2}$ with $E^{2}$ an invertible row and column finite matrix. Note that since no operation was performed on column 0 of $T^{1}$, none was performed on column 0 of $I$. Moreover, rows 0 of $T^{1}$ and $I$ remained unchanged (because of 0 entries), so that $E_{00}^{2}=1$ and the rest of the row 0 and column 0 entries of $E^{2}$ are 0 . Thus the same is true of $G^{2}$, the inverse of $E^{2}$. Since $F=T^{2} G^{2} G^{1}, \mathbf{y}=F \mathbf{x}=$ $T^{2} G^{2}\left(G^{1} \mathbf{x}\right)=T^{2}\left(G^{2} \mathbf{x}^{1}\right)$. Set $\mathbf{x}^{2}=G^{2} \mathbf{x}^{1}$ so that $\mathbf{y}=T^{2} \mathbf{x}^{2}$. Because row 0 of $G^{2}$ is $1,0, \ldots$, it follows that $x_{0}^{2}=x_{0}^{1}$.

Suppose that after $n>1$ iterations, row finite matrices $T^{1}, \ldots, T^{n}$ and invertible row and column finite matrices $E^{1}, \ldots, E^{n}$ with inverses $G^{1}, \ldots, G^{n}$ and $\mathbf{x}^{1}, \ldots, \mathbf{x}^{n} \in \mathbf{P}$ have been obtained, such that

(a) $T_{i j}^{n}=0$ for all $j>i, 0 \leq i \leq n-1$; that is, the matrices $T^{n}$ are becoming increasingly lower triangular;

(b) column $j$ of $T^{n}$ is identical to column $j$ of $T^{n-1}$ for $0 \leq j<n-1$; that is, after the $n$th iteration, rows and columns $<n$ do not change;

(c) $T^{n}=T^{n-1} E^{n}=F E^{1} \cdots E^{n}$;

(d) $E_{i j}^{n}=\delta_{i j}=G_{i j}^{n}, 0 \leq i, j<n-1$; that is, the $E^{n \prime}$ s and their inverses are becoming increasingly diagonal with 1 's down the diagonal, tending toward the identity matrix;

(e) $\mathbf{x}^{n}=G^{n} \mathbf{x}^{n-1}, \mathbf{y}=T^{n} \mathbf{x}^{n}$; that is, $\mathbf{y}$ is always in the image of $T^{n}$;

(f) $x_{i}^{n}=x_{i}^{n-1}, 0 \leq i<n-1$; that is, after the $n$th iteration, the entries $<n$ of the $\mathbf{x}^{n}$ s do not change.

As before, if $T_{n j}^{n}=0$ for all $j \geq n$, simply set $T^{n+1}=T^{n}, E^{n+1}=I$, and $\mathbf{x}^{n+1}=\mathbf{x}^{n}$ and continue. Otherwise, perform column operations on the nonzero entries of the $n$th row in columns $j \geq n$ of $T_{n}$ to obtain a new matrix $T^{n+1}$ in which $T_{n j}^{n+1}=0$ for all $j>n$. Because no operation is performed on columns 0 through $(n-1), T_{i j}^{n+1}=T_{i j}^{n}, i \in \mathbb{N}, j=0, \ldots, n-1$; that is, columns 0 through $(n-1)$ of $T^{n+1}$ are the same as columns 0 through $(n-1)$ of $T^{n}$.

Perform the same column operations on $I$ to produce $E^{n+1}$, so that $T^{n+1}=T^{n} E^{n+1}$ with $E^{n+1}$ an invertible row and column finite matrix having inverse $G^{n+1}$. Note that since 
no operation was performed on columns $0-(n-1)$ of $T^{n}$, none was performed on columns $0-(n-1)$ of $I$. Moreover, rows $0-(n-1)$ of $T^{n}$ were not changed (because of 0 's), and the same is true of $I$ and $G^{n+1}$. (i) As a result, $E_{i j}^{n+1}=\delta_{i j}=G_{i j}^{n+1}, i, j<n$. Since $T^{n+1}=$ $T^{n} E^{n+1}=\left(F E^{1} \cdots E^{n}\right) E^{n+1}, F=T^{n+1} G^{n+1} \cdots G^{1}, \mathbf{y}=F \mathbf{x}=T^{n+1} G^{n+1} \cdots G^{1} \mathbf{x}=T^{n+1}\left(G^{n+1} \mathbf{x}^{n}\right)$. (ii) Set $\mathbf{x}^{n+1}=G^{n+1} \mathbf{x}^{n}$ so that $\mathbf{y}=F^{n+1} \mathbf{x}^{n+1}$. (i) and (ii) imply that $x_{i}^{n+1}=x_{i}^{n}, i=0, \ldots, n-1$.

The Cauchy sequence $\mathbf{x}^{n}$ converges to some $\mathbf{x}^{\prime} \in \mathbf{P}$ because $\mathbf{P}$ is a complete metric space. Since all endomorphisms of $\mathbf{P}$ are continuous, the sequence $T^{n} \mathbf{x}_{j}$ converges to $T^{n}\left(\mathbf{x}^{\prime}\right)$ for each $n$. Because of (b) and (f), the convergence is uniform. The Cauchy sequence of matrices $T^{n}$ thus obtained converges to a lower triangular matrix $T$ and $T \mathbf{x}^{\prime}=\mathbf{y}$ so that $T \mathbf{P} \supseteq F \mathbf{P}$. From (b) it follows that $T \mathbf{P} \subseteq F \mathbf{P}$ and so $T \mathbf{P}=F \mathbf{P}$.

It may, of course, happen that some of the diagonal terms of $T$ are 0 so that the first nonzero entry in a column may be below the diagonal. Should that occur, the first nonzero entry in the next column will be in a lower row. If $T$ has only finitely many nonzero columns, the result is elementary. If all columns of $T$ are nonzero, it is clear that an infinite linear combination of its columns can be $\mathbf{0}$ only if all coefficients are 0 , so that the columns of $T$ form a product basis.

Remark 4.2. From the results in Section 3, a matrix $\in \mathcal{F}$ can be classified as epic or monic if the endomorphism of $\mathbf{P}$ which it induces is epic or monic, respectively. (1) If $F \in \mathcal{F}$ has a 0 row $i$, it cannot be epic because $\mathbf{e}_{i}$ cannot be reached. If it has a 0 column $j$, it cannot be monic because $\mathbf{e}_{j}$ is in the kernel. Here $E=\left\{\mathbf{e}_{i}: i \in \mathbb{N}\right\}$ denotes the usual basis of $\boldsymbol{\Sigma} \subset \mathbf{P}$. (2) Similarly, if a row (column) of $F$ is a multiple of another row (column), then $F$ cannot be epic (monic). (3) $T \in \tau$ is invertible ( 2 sided) over the rationals if and only if each diagonal entry is nonzero [5, pages 19-20 ]. (4) $T$ is integrally invertible if and only if each diagonal entry $= \pm 1$.

Lemma 4.3. $T \in \tau$ is epic if and only if each diagonal entry is \pm 1 .

Proof. If each diagonal entry of $T$ is \pm 1 , then $T \in \mathcal{G} \perp$ and so is epic. Conversely let $\mathbf{y}=$ $\left(y_{0}, y_{1}, \ldots\right)^{t}$ be a nonzero element of $\mathbf{P}$. To solve $T \mathbf{x}=T\left(x_{0}, x_{1}, \ldots\right)^{t}=\mathbf{y}$, induct on $n$. Let $T^{n}$ denote the finite matrix $\left(T_{i j}: i, j=0,1, \ldots, n\right)$. If $n=0$ and $T_{00} \neq \pm 1$, then $T_{00} x_{0}=y_{0}$ is not solvable in general. If $T_{00}= \pm 1$, then $x_{0}$ is uniquely determined. Suppose that the diagonals $T_{k k}= \pm 1$ for $k=0,1, \ldots, n$, and suppose further that $T^{n}\left(x_{0}, \ldots, x_{n}\right)^{t}=\left(y_{0}, \ldots, y_{n}\right)^{t}$ is uniquely solvable, $\left(x_{0}, \ldots, x_{n}\right)^{t}=S^{n}\left(y_{0}, \ldots, y_{n}\right)^{t}$, where $S^{n}$ denotes the inverse of $T^{n}$. Express

$$
T^{n+1}=\left(\begin{array}{ccc}
T^{n} & & 0 \\
T_{n+1,0} & \cdots & T_{n+1, n+1}
\end{array}\right)
$$

so that

$$
T^{n+1}\left(x_{0}, \ldots, x_{n}, x_{n+1}\right)^{t}=\left(\begin{array}{c}
T^{n}\left(x_{0}, \ldots, x_{n}\right)^{t} \\
\sum_{j=0}^{j=n+1} T_{n+1, j} x_{j}
\end{array}\right)
$$

with some abuse of notation for the sake of simplicity. Now by the induction hypothesis, $\left(x_{0}, \ldots, x_{n}\right)^{t}=S^{n}\left(y_{0}, \ldots, y_{n}\right)^{t}$ uniquely. Thus the only solution to $\sum_{j=0}^{j=n+1} T_{n+1, j} x_{j}=y_{n+1}$ must 
come from $T_{n+1, n+1} x_{n+1}=y_{n+1}-\sum_{j=0}^{j=n} T_{n+1, j} x_{j}$, which is impossible in general unless $T_{n+1, n+1}=$ \pm 1 . follows.

Invertible lower triangular infinite integral matrices may therefore be characterized as

Theorem 4.4. For $T \in \tau$ the following are equivalent.

(1) $T$ is epic.

(2) $T_{k k}= \pm 1$ for all $k \in \mathbb{N}$.

(3) $T \in \mathcal{G} \mathcal{L}$.

By now the following result is clear.

Corollary 4.5. $T \in \tau$ is monic if and only if $T_{k k} \neq 0$ for all $k \in \mathbb{N}$.

\section{Infinite Matrix Examples and Counterexamples}

The next examples and counterexamples serve to justify some of the assumptions about matrix rings made in this paper. They illustrate some of the pitfalls and limitations of working with infinite matrices and further illustrate results from Section 4. They are not new but continue to be discussed in various contexts: [9, section 2]; [10, page 437]; [7, pages 189-191].

As before, the elements of $\mathbf{P}$ are viewed as column vectors and $E=\left\{\mathbf{e}_{j}: j \in \mathbb{N}\right\}$ denotes the usual basis of $\boldsymbol{\Sigma} \subset \mathbf{P} ; E$ is also the standard product basis of $\mathbf{P}$. The same symbol $E$ is used for the matrix with the $\mathbf{e}_{j}$ as columns, that is, the identity matrix. The same liberty is taken with other bases and their matrices.

Example 5.1. $E_{1}=\left\{\mathbf{e}_{0}+\mathbf{e}_{1}+\cdots+\mathbf{e}_{i}: i \in \mathbb{N}\right\}$ also is a basis of $\boldsymbol{\Sigma}$, having matrix

$$
E_{1}=\left(\begin{array}{ccccc}
1 & 1 & 1 & 1 & \cdots \\
0 & 1 & 1 & 1 & \ldots \\
0 & 0 & 1 & 1 & \ldots \\
0 & 0 & 0 & 1 & \cdots \\
\vdots & \vdots & \vdots & \vdots & \ddots
\end{array}\right) .
$$

Because the matrix is not row finite, $E_{1}$ cannot be a product basis of $\mathbf{P}$.

Example 5.2. $E_{2}=\left\{\mathbf{e}_{0}, \mathbf{e}_{i}-\mathbf{e}_{i-1}: i \in \mathbb{N}^{+}\right\}$also is a basis of $\boldsymbol{\Sigma}$, having matrix

$$
E_{2}=\left(\begin{array}{ccccc}
1 & -1 & 0 & 0 & \cdots \\
0 & 1 & -1 & 0 & \cdots \\
0 & 0 & 1 & -1 & \cdots \\
0 & 0 & 0 & 1 & \cdots \\
\vdots & \vdots & \vdots & \vdots & \ddots
\end{array}\right)
$$

Note that $E_{2}$ is not lower triangular. 
Example 5.3. Let $\mathbf{1} \in \mathbf{P}$ denote the column vector having all entries $=1$ and let $E_{3}=\left\{\mathbf{e}_{0}+\mathbf{e}_{2}+\right.$ $\left.\cdots+\mathbf{e}_{i-1}-\mathbf{1}: i \in \mathbb{N}^{+}\right\}$. Note that $E_{3}$ is not a basis of $\boldsymbol{\Sigma}$; indeed, no element is even contained in $\boldsymbol{\Sigma}$. The accompanying lower triangular matrix is

$$
E_{3}=\left(\begin{array}{ccccc}
0 & 0 & 0 & 0 & \cdots \\
-1 & 0 & 0 & 0 & \cdots \\
-1 & -1 & 0 & 0 & \cdots \\
-1 & -1 & -1 & 0 & \cdots \\
\vdots & \vdots & \vdots & \vdots & \ddots
\end{array}\right) .
$$

The following relationships hold:

$$
E_{1} E_{2}=E=E_{2} E_{1}, \quad E_{2} E_{3}=E
$$

Observe that, although both $E_{2}$ and $E_{3}$ are row finite and even pure independent, $E_{2}$ is not monic (1 is in the kernel), and $E_{3}$ is not epic ( $\mathbf{e}_{0}$ is not attained). In particular, $\left(E_{1} E_{2}\right) \mathbf{1}=$ $E \mathbf{1}=\mathbf{1}$, but $E_{1}\left(E_{2} \mathbf{1}\right)=E_{1}(\mathbf{0})=\mathbf{0}$, so that multiplication is not associative. Despite the fact that $E_{2} \mathbf{P}=\mathbf{P}, E_{2}$ nevertheless cannot serve as a product basis for $\mathbf{P}$. The problem, of course, is that $E_{2}$ is not invertible in $\mathscr{F}$. Restriction to multiplicatively associative matrix rings helps to eliminate much of this infinite matrix pathology [5, pages 19-22].

In the proof of Theorem 4.1, although the columns of the resulting lower triangular matrix $T$ obviously are independent, $T$ nevertheless may be singular, as illustrated by $E_{3}$ above. Moreover, it may not be the case that the products $E^{1} \cdots E^{n}$ in that proof will converge to a row finite matrix, as further illustrated by $E_{2}$. Even though $E_{2}$ itself is not monic, the resulting lower triangular matrix $E$, which results from column operations on $E_{2}$, is monic.

\section{Fuchs' Lemma 95.1 Redux}

Products in $\mathbf{P}$ were introduced in [2] by beginning with a countable subset $\left\{\mathbf{x}_{n}: n \in \mathbb{N}\right\} \subset \mathbf{P}$, with no condition other than that all sums of the form $\mathbf{x}=\sum_{n=0}^{\infty} s_{n} \mathbf{x}_{n}, s_{n} \in \mathbb{Z}$, are well defined. As mentioned, this means that the infinite matrix with columns $\mathbf{x}_{n}$ must be row finite. Lemma 95.1 then was stated as follows in [2].

Let $X$ be a product in $\mathbf{P}$. There are elements $\mathbf{a}_{n} \in \mathbf{P}$ and integers $k_{n}(n \in \mathbb{N})$ such that $\mathbf{P}=\prod_{n=0}^{\infty}\left\langle\mathbf{a}_{n}\right\rangle$ and $X=\prod_{n=0}^{\infty}\left\langle k_{n} \mathbf{a}_{n}\right\rangle$ where $(\bullet) k_{n} \mid k_{n+i}$ if $k_{n} \neq 0$ for all $n, i \geq 0$.

The goal was to establish a product analog of the well-known result for stacked bases of free groups of finite rank [1, Lemma 15.4]. However, a counterexample has been produced [11].

Perhaps another approach is to utilize the weaker definition of stacked bases found in [12], which does not require divisibility as in $(\bullet)$. From Theorem 4.1 , there would be no loss of generality in assuming that the $\mathbf{x}_{n} \in \mathbf{P}$ form a product basis and a lower triangular matrix.

Lemma 6.1. Let $\prod_{n=0}^{\infty}\left\langle\mathbf{x}_{n}\right\rangle$ be a product of infinite rank in $\mathbf{P}$, let $S=\sum_{n=0}^{\infty}\left\langle\mathbf{x}_{n}\right\rangle$, and let $S_{*}$ be the pure subgroup which the $\mathbf{x}_{n}$ generate. Then there exist a product basis of $\mathbf{P}=\prod_{n=0}^{\infty}\left\langle\mathbf{a}_{n}\right\rangle$ and positive 
integers $k_{n}(n \in \mathbb{N})$ such that $\prod_{n=0}^{\infty}\left\langle k_{n} \mathbf{a}_{n}\right\rangle=\prod_{n=0}^{\infty}\left\langle\mathbf{x}_{n}\right\rangle$ if and only if there exist stacked bases of $S$ and $S_{*}$ such that the matrix $F_{*}$ formed by the basis of $S_{*}$ is invertible and the matrix $F$ formed by the basis of $S$ satisfies $F \mathbf{P}=\prod_{n=0}^{\infty}\left\langle\mathbf{x}_{n}\right\rangle$.

Proof. If $\left\{\mathbf{a}_{n}: n \in \mathbb{N}\right\}$ is a product basis of $\mathbf{P}$, then the $\mathbf{a}_{n}$ 's are pure independent. If $\left\{k_{n}\right.$ : $n \in \mathbb{N})\}$ are positive integers, then $\prod_{n=0}^{\infty}\left\langle k_{n} \mathbf{a}_{n}\right\rangle$ is a product in $\mathbf{P}$ and if $S=\oplus_{n=0}^{\infty}\left\langle k_{n} \mathbf{a}_{n}\right\rangle$, then $S_{*}=\oplus_{n=0}^{\infty}\left\langle\mathbf{a}_{n}\right\rangle$ so that $\left\{k_{n} \mathbf{a}_{n}: n \in \mathbb{N}\right\}$ and $\left\{\mathbf{a}_{n}: n \in \mathbb{N}\right\}$ are stacked bases for the respective subgroups of $\mathbf{P}$. The matrix formed by the $\mathbf{a}_{n}$ 's is in $\mathcal{G}_{\mathcal{L}} \mathcal{L}$ by Corollary 3.3; the matrix $F$ formed by the $\mathbf{x}_{n}=k_{n} \mathbf{a}_{n}$ certainly satisfies $F \mathbf{P}=\prod_{n=0}^{\infty}\left\langle\mathbf{x}_{n}\right\rangle$.

Conversely, let $\prod_{n=0}^{\infty}\left\langle\mathbf{x}_{n}\right\rangle$ be a product in $\mathbf{P}$, let $S$ be the subgroup of $\mathbf{P}$ which the $\mathbf{x}_{n}$ 's generate, and let $S_{*}$ be the pure subgroup which they generate. Suppose there are stacked bases $\left\{k_{n} \mathbf{a}_{n}: k_{n}, n \in \mathbb{N}\right\}$ of $S$ and $\left\{\mathbf{a}_{n}: n \in \mathbb{N}\right\}$ of $S_{*} ;$ because $S_{*} / S$ is torsion, all $k_{n}$ 's must be positive. Suppose further that the matrix formed by the $\mathbf{a}_{n}{ }^{\prime}$ s is invertible; then the $\mathbf{a}_{n}{ }^{\prime}$ s are a product basis of $\mathbf{P}$ and $\prod_{n=0}^{\infty}\left\langle k_{n} \mathbf{a}_{n}\right\rangle$ is a product in $\mathbf{P}$. Finally, if the matrix $F$ formed by the $k_{n} \mathbf{a}_{n}{ }^{\prime}$ 's satisfies $F \mathbf{P}=\prod_{n=0}^{\infty}\left\langle\mathbf{x}_{n}\right\rangle$, the proof is concluded.

Remark 6.2. (1) If $\prod_{n=0}^{\infty}\left\langle\mathbf{x}_{n}\right\rangle$ is a product in $\mathbf{P}$ and $\left\{\mathbf{a}_{n}: n \in \mathbb{N}\right\}$ is a basis of the subgroup generated by the $\mathbf{x}_{n}{ }^{\prime}$ s, the matrix formed by the $\mathbf{a}_{n}$ 's need not be row finite. (2) As previously noted, the lower triangular matrix $T$, obtained from the column operations in Theorem 4.1, may be singular. However, Nunke [8, page 199] has shown that every endomorphic image of $\mathbf{P}$ is either free of finite rank or isomorphic to $\mathbf{P}$ itself. In the latter case, there then exists monic $F \in \mathcal{F}$ satisfying $F \mathbf{P}=T \mathbf{P}$. Column operations will, of course, reveal matrix rank, finite or infinite.

All products in $\mathbf{P}$ may therefore be characterized as follows.

Theorem 6.3. Every product in $\mathbf{P}$ is generated by a lower triangular, infinite, integral matrix, the columns of which form a product basis and the rank of which determines whether the product is free of finite rank or isomorphic to $\mathbf{P}$.

\section{Generalizations}

The author's initial draft of this paper was done for higher dimensions, along the lines of [13]. At the referee's suggestion, matrix dimension has been restricted to 2 , to improve readability.

\section{References}

[1] L. Fuchs, Infinite Abelian Groups. Vol. I, vol. 36 of Pure and Applied Mathematics, Academic Press, New York, NY, USA, 1970.

[2] L. Fuchs, Infinite Abelian Groups. Vol. II, vol. 36 of Pure and Applied Mathematics, Academic Press, New York, NY, USA, 1973.

[3] R. Baer, "Abelian groups without elements of finite order," Duke Mathematical Journal, vol. 3, no. 1, pp. 68-122, 1937.

[4] E. Specker, "Additive Gruppen von Folgen ganzer Zahlen," Portugaliae Mathematica, vol. 9, pp. 131140, 1950.

[5] R. G. Cooke, Infinite Matrices and Sequence Spaces, McMillan, London, UK, 1950.

[6] A. Blass and J. Irwin, "Free subgroups of the Baer-Specker group," Communications in Algebra, vol. 29, no. 12, pp. 5769-5794, 2001.

[7] A. R. Blass and J. Irwin, "On the group of eventually divisible integer sequences," in Abelian Groups and Modules (Colorado Springs, CO, 1995), D. M. Arnold and K. M. Rangaswamy, Eds., vol. 182 of 
Lecture Notes in Pure and Applied Mathematics, pp. 181-192, Marcel Dekker, New York, NY, USA, 1996.

[8] A. R. Blass and J. Irwin, "Baer meets Baire: applications of category arguments and descriptive set theory to $\mathbb{Z}^{\aleph_{0}}$," in Abelian Groups and Modules (Colorado Springs, CO, 1995), D. M. Arnold and K. M. Rangaswamy, Eds., vol. 182 of Lecture Notes in Pure and Applied Mathematics, pp. 193-202, Marcel Dekker, New York, NY, USA, 1996.

[9] Z. Bartosiewicz and D. Mozyrska, "Observability of row finite countable systems of linear differential equations," in Proceedings of the 16th IFAC World Congress, Prague, Czech Republic, July 2005.

[10] V. Camillo, F. J. Costa-Cano, and J. J. Simon, "Relating properties of a ring and its ring of row and column finite matrices," Journal of Algebra, vol. 244, no. 2, pp. 435-449, 2001.

[11] B. Goldsmith, "A note on products of infinite cyclic groups," Rendiconti del Seminario Matematico della Università di Padova, vol. 64, pp. 243-246, 1981.

[12] J. M. Cohen and H. Gluck, "Stacked bases for modules over principal ideal domains," Journal of Algebra, vol. 14, pp. 493-505, 1970.

[13] E. F. Cornelius, Jr. and P. Schultz, "Multinomial points," Houston Journal of Mathematics, vol. 34, no. 3, pp. 661-676, 2008. 


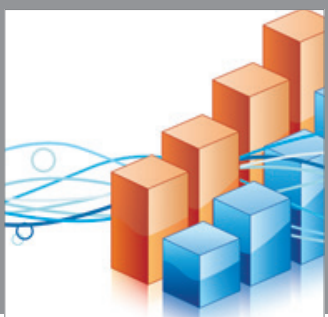

Advances in

Operations Research

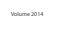

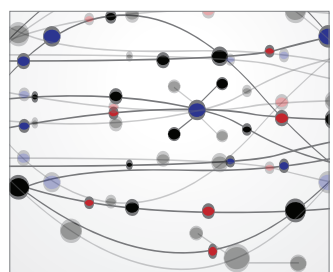

\section{The Scientific} World Journal
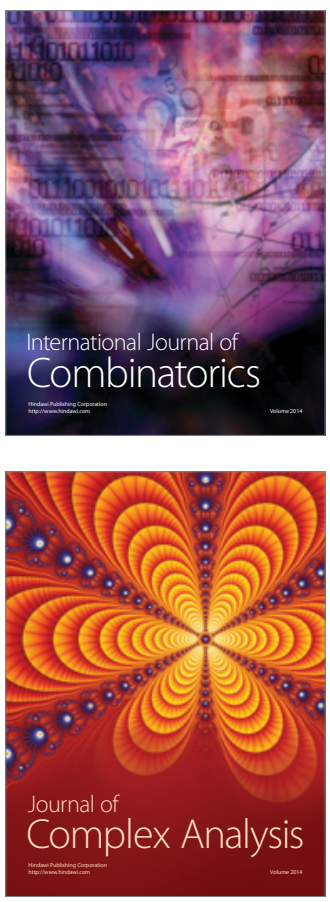

International Journal of

Mathematics and

Mathematical

Sciences
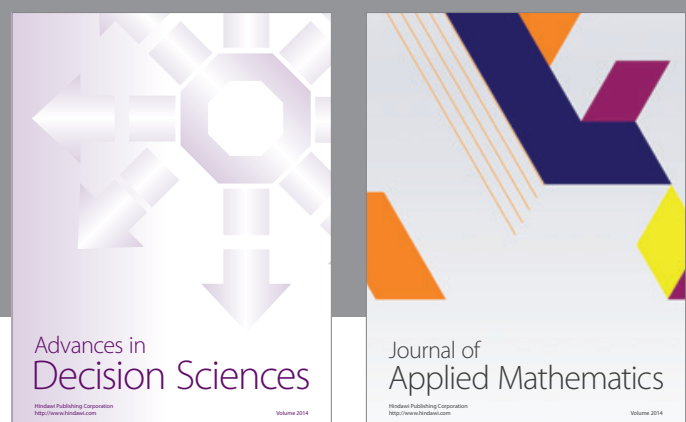

Journal of

Applied Mathematics
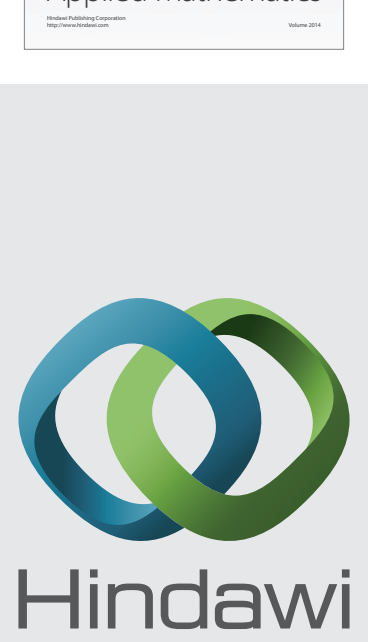

Submit your manuscripts at http://www.hindawi.com
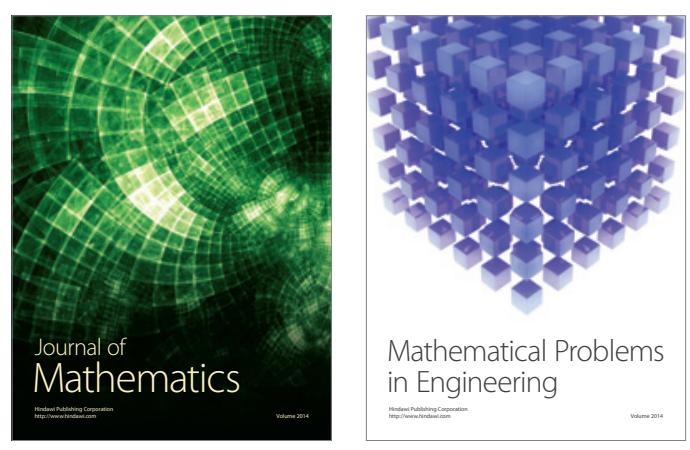

Mathematical Problems in Engineering
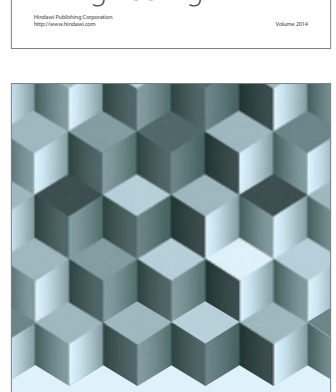

Journal of

Function Spaces
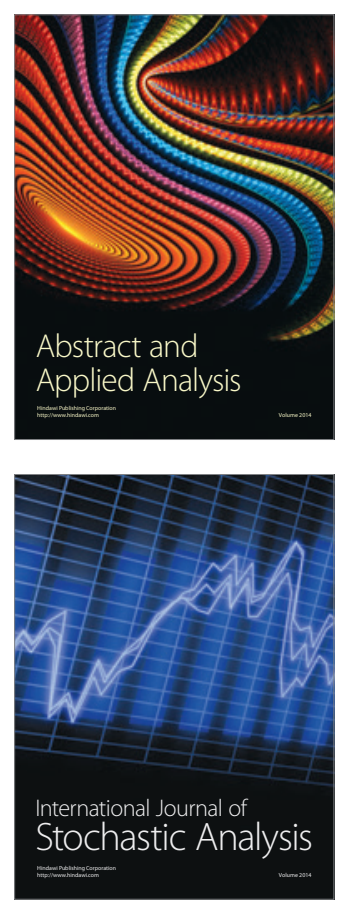

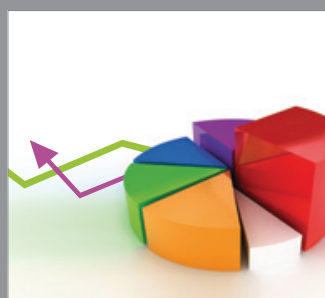

ournal of

Probability and Statistics

Promensencen
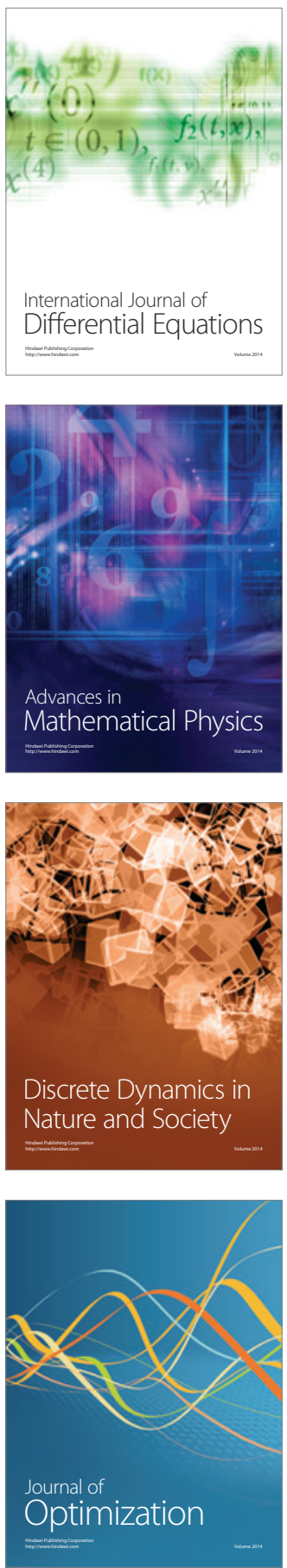